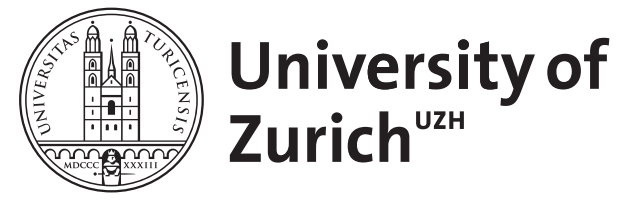

"Tailored-to-You": Public Engagement and the Political Legitimation of Precision Medicine

\author{
Blasimme, Alessandro ; Vayena, Effy
}

DOI: https://doi.org/10.1353/pbm.2017.0002

Posted at the Zurich Open Repository and Archive, University of Zurich ZORA URL: https://doi.org/10.5167/uzh-135639

Journal Article

Published Version

Originally published at:

Blasimme, Alessandro; Vayena, Effy (2016). "Tailored-to-You": Public Engagement and the Political Legitimation of Precision Medicine. Perspectives in Biology and Medicine, 59(2):172-188.

DOI: https://doi.org/10.1353/pbm.2017.0002 


\section{PROJECT MUSE}

"Tailored-to-You": Public Engagement and the Political Legitimation of Precision Medicine

Alessandro Blasimme, Effy Vayena

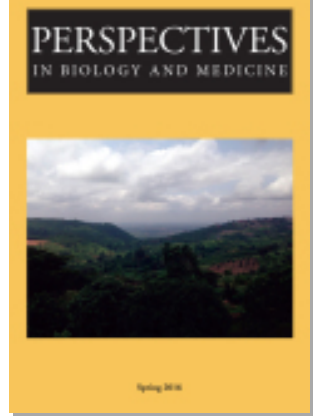

Published by Johns Hopkins University Press

DOI: https://doi.org/10.1353/pbm.2017.0002

Perspectives in Biology and Medicine, Volume 59, Number 2, Spring 2016, pp. 172-188 (Article)

Dor: hitps//doi.org/10.1353/pbm.2017.002

$\Rightarrow$ For additional information about this article https://muse.jhu.edu/article/648044 
“TA I LORED-TO-YOU”

\title{
public engagement and the political legitimation of precision medicine
}

\section{Alessandro Blasimme AND EFFy VAyena}

\begin{abstract}
With the launch of the Precision Medicine Initiative (PMI) in January 2015, the White House granted a high degree of federal support to an emerging biomedical paradigm. What explains this level of political recognition? Based on literature and policy analysis, we reconstruct the scientific and the legislative paths that led to the political endorsement of precision medicine. First, we describe the proliferation of personalized approaches to therapy ignited by the discovery of hemoprotein cytochrome P450 polymorphism in 1988. Then, we analyze the legislative history of precision medicine, from the unsuccessful introduction of Genomics and Personalized Medicine Acts in the second half of the last decade, to the highly acclaimed PMI. However, scientific progress and political contingency alone do not explain the upheaval of precision medicine as an institutionally supported initiative. On examination, the launch of a Precision Medicine Research Cohort and the incorporation of a participatory ethos into the fabric of the PMI proved to be crucial determinants of the political support for precision medicine. Weaving together the scientific and legislative antecedents of precision medicine, we illuminate how the mutual constitution of science and social order generates political recognition for innovative biomedical paradigms.
\end{abstract}

\footnotetext{
Health Ethics and Policy Lab, Institute of Epidemiology Biostatistics and Prevention, University of Zurich, Switzerland.

Correspondence: Effy Vayena, Health Ethics and Policy Lab, EBPI, University of Zurich, Hirschengraben 84, 8001 Zurich, Switzerland.

E-mail: effy.vayena@uzh.ch.
}

Perspectives in Biology and Medicine, volume 59, number 2 (spring 2016): 172-188.

(C) 2017 by Johns Hopkins University Press 
C ome patients tolerate a given drug well, without adverse reactions. For othSers, though, an identical dose of the same medication can have toxic effects. Moreover, while a drug can be effective at relieving symptoms for some patients, it may fail to do the same for others suffering with the same disease. With such variability in treatment responses, tailoring medical interventions to individual patients has long been an aspiration of medicine. Until recently, however, medicine lacked a clear understanding of the biological reasons for human variation in drug response. Attempting to adjust treatment to the individual patient, physicians have relied primarily on direct observation, trial-and-error, and, ultimately, on their own clinical judgment.

In the late 1980s, analyses of the genetic bases of individual variation in drug response propelled unprecedented scientific interest in the prospects of developing more accurate, patient-tailored preventive, diagnostic, and therapeutic interventions. During the next two decades, the label "personalized medicine" was used to designate a bundle of emerging approaches based on technological and scientific advancements in fields such as genetics, genomics, bioinformatics, and targeted pharmacology. The promoters of personalized medicine have described these approaches as a novel medical project aimed at providing "each patient with the right drug at the right dose at the right time" (Hamburg and Collins 2010). To be sure, some commentators have been skeptical of the assertion that the concept of personalized medicine is something new (Steele 2009). Nevertheless, promises of an imminent clinical revolution have driven considerable interest. Recently, the promise of personalized health care has acquired strong political support, most notably evidenced by the launch of the Precision Medicine Initiative (PMI) by President Barack Obama in January 2015, and its associated national research cohort comprised of 1 million Americans and run by the National Institutes of Health (NIH).

Precision medicine is the latest branding of a family of approaches-including pharmacogenetics, pharmacogenomics, P4 medicine, and personalized medicine-all aimed at tailoring therapy to patients. The first clinical successes in this area date back to the late 1990 s, with the development of the first targeted therapies, such as Herceptin for HER2-positive breast cancer, approved by the Food and Drug Administration (FDA) in 1998. It is notable, however, that it took another 20 years after these success stories for personalized medicine to be endorsed politically. Our aim is not to assess whether that support is warranted, let alone to promote the claim that precision medicine will bring about a major shift in medical research and clinical care. Rather, we aim to examine the organizational and ethical elements that were mobilized to win political support for precision medicine after a series of failed attempts.

To explain the political uptake of precision medicine, we reviewed both its scientific and political antecedents. In particular, we reconstructed the basic features of four approaches developed after the discovery of hemoprotein cytochrome 
P450 polymorphism in 1988, a scientific milestone in the understanding of human variability in drug metabolism: pharmacogenetics, pharmacogenomics, personalized medicine and P4 medicine (see Table 1). We drew from the most cited papers describing each of these approaches as the basis for our content analysis. ${ }^{1}$ We then compared the results of this analysis with how personalized medicine is described and imagined in political discourse. To this aim, we examined four legislative acts that were unsuccessfully introduced in the Congress of the United States between 2006 and 2010 and intended to ensure support for genomics and personalized medicine. Finally, we compared the imagined features of personalized medicine in those policy documents with the present framing of the White House Precision Medicine Initiative (PMI). ${ }^{2}$ Through this process, we were able to trace the emergence of novel elements in the framing of personalized medicine that made this iteration politically more palatable.

\section{Genes, DRugs, ANd THE Puzzle of Individual VARIATION}

Individual variability in response to drugs has long been a vexing issue in medicine and pharmacology. However, the realization that this issue could be illuminated in terms of genetics, dates back only to the late 1950s, when Fredrich Vogel introduced the term pharmacogenetics to designate a branch of medicine focused on identifying the genetic determinants of variation in drug response (Jones 2013).

This new field did not generate much clinical interest until the 1980s, when allelic variation in cytochrome P450 (a major genetic locus encoding for multiple variants of crucial receptors for drug metabolism) started to be extensively characterized (Gonzalez 1988). Following this discovery, multiple research efforts in both the public and private sectors explored the possibility of tailoring treatments to the specificities of each patient.

The term pharmacogenetics gained traction in the early 1990s, as the relationship among genes, drugs, and disease outcomes attracted increasing scientific attention. Pharmacogentics generally refers to two distinct but interrelated endeavors: clarifying the genetic bases of variability in adverse drug reactions and drug response, and understanding the genetic components of human disease in order to isolate novel pharmacological targets (Meyer 2000; Roses 2000, 2004). The first strand of

\footnotetext{
${ }^{1}$ For each paradigm we have searched the Web of Science for papers featuring the name of the paradigm in the title field. (For P4 medicine we had to query "P4" AND "medicine," and we also queried "P4 medicine" in the topic field and generated a list with the first 10 items for number of citations from each search.) The period was 1988-2016. Our inclusion criteria were as follows: only papers in English appearing in the first 50 hits of the list (from most to less cited); only unspecific papers (with no mention of specific genetic loci or disease in the title-but papers with only "cancer" were retained); and at least one citation. We retained the first 10 unspecific papers (from most to less cited); irrelevant papers were cleaned up manually.

${ }^{2}$ For our analysis of the PMI, we have used the following sources: PMI Working Group 2015; White House 2015a, 2015b, 2015c, 2016.
} 
Table 1 Publication Trends for Pharmacogenetics, Pharmacogenomics, and Personalized Medicine

\begin{tabular}{lrrrr}
\hline Period & Pharmacogenetics & Pharmacogenomics & $\begin{array}{c}\text { Personalized } \\
\text { medicine }\end{array}$ & $\begin{array}{c}\text { All medical } \\
\text { articles }\end{array}$ \\
\hline $\mathbf{2 0 0 1 - 2 0 0 5}$ & $837(0.15 \%)$ & $724(0.13 \%)$ & $202(0.03 \%)$ & 532,348 \\
$\mathbf{2 0 0 6}-\mathbf{2 0 1 0}$ & $1,358(0.19 \%)$ & $1,098(0.15 \%)$ & $1,249(0.17 \%)$ & $708,900[1.3]$ \\
$\mathbf{2 0 1 1 - 2 0 1 5}$ & $1,918(0.16 \%)$ & $1,680(0.14 \%)$ & $4,764(0.41 \%)$ & $1,154,041[1.6]$
\end{tabular}

$\star$ Absolute numbers obtained by querying for the word medical in any field in each time period. Numbers in square brackets represent the fold change increase with respect to the previous period.

Data show the number of PubMed publications featuring the words pharmacogenetics, pharmacogenomics, and personalized medicine (or personalised medicine) in either title or abstract. We report absolute numbers for each period and, in brackets, the percentage these numbers represent of the overall number of medical articles in the same period. Publication trends show that "personalized medicine" enjoyed a more rapid growth (from $0.17 \%$ to $0.41 \%$ of all published medical articles) than the two other paradigms between 2011 and 2015 . Between 2001 and 2015, a total of 46 publications mention "P4 medicine" in their title or abstract (data not included in the table).

pharmacogenetics offered insight into the genetics behind variability in pharmacokinetics and pharmacodynamics, and thus promised to lead to improved drug safety and efficacy (Meyer 2000). The second strand, instead, aimed at developing more effective drugs based on the identification of previously unknown molecular targets. In both cases, however, emphasis is on making and administering drugs that are tailored to the individual genetic features of patients, thus pushing medicine towards a more personalized approach. The language of personalization is already present in some influential publications of this early period (Roses 2000), but at the turn of the century, the term pharmacogenetics was much more commonly used than "personalized medicine."

More specifically, in the case of drug response, the discovery of genetic factors influencing how a patient metabolizes a given drug led to the realization that patients can be stratified according to their genotype and assigned to genotype-specific pharmacological regimes (Gardiner and Begg 2006; Meyer 2000; Roses 2004). This conceptual shift played out even more prominently in the development of drugs targeted to the varying genetic characteristics of human diseases. ${ }^{3}$ Novel disease taxonomies were created, as previously unified conditions were specified as sub-diseases, each bearing distinct genetic characteristics. Following this strategy of stratification (rather than a language of personalization), scientists and companies working in pharmacogenetics were directed their efforts towards developing small molecules capable of modifying a growing number of new tar-

\footnotetext{
${ }^{3}$ In PubMed publications, the term stratification does not appear in association with "drug response" before 1999 (in titles and abstracts). This indicates that the concept of stratification in pharmacology represents a novelty in this field.
} 
gets. With this reclassification, patients wound up in smaller disease-groups, receiving more tailored and, ideally, more effective drugs that matched the precise genetic features of their own diseases. In breast cancer, for example, the realization that a sub-group of patients overexpresses a specific protein (HER2) led to the development of trastuzumab, a monoclonal antibody eventually approved by the FDA in 1998 with the commercial name Herceptin. This approval is generally considered a topical success story for pharmacogenetics. Moreover, other drugs approved around the same years count as success stories for the second strand of pharmacogenetics, focused on targeting specifically defined components of molecular pathways involved in disease onset and progression. Prominent examples include imatinib for chronic myeloid leukemia, approved in 2001 as Gleevec, which was later extended to treat gastrointestinal stromal tumors (where it blocks the c-Kit cascade).

\section{A Genomic Turn}

In 2000, the prospects of pharmacogenetics were galvanized by the completion of the Human Genome Project (HGP), and then the first draft of the entire sequence of human DNA. Soon after came rapid progress in the development of faster and cheaper sequencing technologies, gradually leading to the introduction of variants for the word pharmacogenetics, terms such as "pharmacogenomics" and "genomic medicine." Obviously, the advantage of genome sequencing compared to more traditional genotyping at specific loci is its ability to capture a wider array of variations, including not only naturally occurring polymorphism and acquired mutations at the level of DNA, but also differences in gene expression that can affect drug metabolism and entire disease-altered molecular pathways (Evans and Relling 1999, 2004).

Genomics, then, promises to identify a spectrum of disease-related alterations as targets for new drugs. Moreover, with pharmacogenomics, the identification of clinically relevant biomarkers (that is, biological markers, like cell surface receptors, or genetic markers, such as single nucleotides polymorphisms) nurtured the expectation that more individualized treatments could soon be discovered. ${ }^{4}$ The value of pharmacogenomic biomarkers lies in their ability to inform therapeutic decisions by predicting mechanisms of action, clinical response, adverse side effects, and genotype-specific dosing for patients carrying the biomarker. Currently, the list of FDA-approved drugs having a pharmacogenomic biomarker information in their label includes 163 drugs, signaling a substantial translational output (FDA 2016).

${ }^{4}$ The NIH Biomarkers Definition Working Group (2001) specifies that the word biomarker should be used to designate "A characteristic that is objectively measured and evaluated as an indicator of normal biological processes, pathogenic processes, or pharmacologic responses to a therapeutic intervention." 


\section{Towards Personalized Medicine}

Around 2005, the term "personalized medicine" started enjoying widespread circulation. Generally, it designates health care “informed by each person's unique clinical, genetic, genomic, and environmental information" (Ginsburg and Willard 2009). The actual implementation of personalized medicine thus depends on the convergence of both data and expertise from different disciplinary ambits. Nevertheless, genomic data and biomarkers feature as focal epistemic drivers in most definitions of personalized medicine (Whirl-Carrillo et al. 2012). Most importantly, this paradigm promotes a comprehensive approach to health care, spanning health monitoring, disease prevention, health promotion, and clinical care. Also interestingly, personalized medicine is often associated with a narrative of optimization of diagnostic and therapeutic interventions and the customization of patient care.

Popularized by the idea of "steering the patient to the right drug at the right dose at the right time" (Hamburg and Collins 2010), personalized medicine soon appeared to be more aspirational than originally believed. In a much cited Perspectives piece in the New England Journal of Medicine, the leadership of the NIH and the FDA emphasized that, despite advances in knowledge and technology, personalized medicine would not materialize on its own without dedicated organizational input. Advances in basic science, translational science, and regulatory science, the authors stressed, must occur to create the conditions for personalized medicine to develop (Hamburg and Collins 2010). Other influential papers also highlight impediments - both scientific and regulatory - to the effective translation of knowledge into personalized clinical applications, insisting that expected advances may not be right around the corner (Chin, Andersen, and Futreal 2011; Ginsburg and McCarthy 2001; Ginsburg and Willard 2009). Even so, success stories in patient-tailored therapy kept the hope alive for a bright future for personalized medicine.

During this same time, the availability of comprehensive data sets prompted interest in a systems-level understanding of biology (Weston and Hood 2004). This approach is based on the elucidation of genetic networks (including both genegene and gene-protein interaction) and biochemical pathways, now enabled by rapidly accruing genomic data. By modeling the systemic properties of biological phenomena, this field of inquiry holds promise of fully exploiting information gained in genome sequencing. Systems biology aims to understand what ensures the robustness, or the homeostatic stability, of a given biological phenomenon and what, on the flip side, leads to its pathological perturbation. The emerging model of system biology gained widespread recognition by the mid-2000s as an approach that, integrating "wet" experimental data and "dry" computational methods, could find relevant application in clinical medicine (Kitano 2002). In particular, identifying disease-perturbed networks in individual patients came to be seen as a proxy for more personalized therapeutic and preventive strategies (Flores et al. 2013). 
Interestingly, among the most-cited papers developing the concept of personalized medicine, this model for personalized medicine is often woven together with that of systems biology (Nicholson 2006; Weston and Hood 2004), each concept leaning on the other for its own articulation.

\section{P4 Medicine: Patients as Partners in Research}

A 2011 paper by Leroy Hood and Stephen Friend introduced the notion of P4 medicine. P4 medicine served as a placeholder for what was to become a more comprehensive approach based on four pillars: prediction, prevention, personalization, and participation. This model focused attention on the emerging conjugation of genomics, computation, and clinical practice. Although the P4 model does not figure largely in major policy initiatives - such as the Stratified Medicine Programme in the United Kingdom (2013) and the PMI in the United States (2015) - it played an important role in the subsequent broadened articulation of personalized medicine. Hood and colleagues argued for an already ongoing transformative shift in medicine, from treating disease in a reactive fashion to maximizing well-being in a proactive way.

This shift is understood as both a technical and a societal revolution. To accomplish the shift, it is argued, medicine should rely on a variety of data, including not only genetic, but also epigenetic, transcriptomic, proteomic, and metabolomic data, as well phenotypic measurements of all sorts. Such comprehensive data sets would enable the construction of predictive models measuring individual baseline wellness, and thus able to predict transition to disease. Combined with efforts aimed at the stratification of diseases into increasingly precise subtypes, these models are supposed to lead to the personalization of treatment to the individual characteristics of each patient. The third pillar of $\mathrm{P} 4$ medicine, prevention, refers to the ability to target "disease-perturbed molecular networks [in order] to deter or stop disease progression" (Hood and Friend 2011). The fourth pillar of the model, participation, has more to do with the societal implementation of the others. In this area, the authors propose moving beyond the current model of clinical trial participation and embracing what they call "citizen-driven trials," in which patients become partners of science on the road to personalized medicine. In this model, research takes place by directly crowd-sourcing patients who have an interest in participating, as opposed to conventional enrollment methods. The rationale for distributed, crowd-sourced trial networks stems from the need to pull data from cohorts much larger than those accessible though a clinical trial, enabling researchers to uncover sets of molecular alterations that can drive clinical stratification. An obvious corollary to this public engagement turn is the willingness of participants to share samples, biological data, phenotypic measurements, and medical health records, possibly in a much more extensive way than is currently allowed under the Common Rule. However, the generation and collection of 
such extensive amounts of data, the authors noted, raises major ethical issues with respect to privacy, data security, and ownership.

Despite the fact that its characteristic emphasis on systems medicine has not become mainstream in current articulations of precision medicine, the $\mathrm{P} 4$ model has crystallized the link between personalized approaches to medicine and the ethos of participation. This feature is still central to what is now called precision medicine.

\section{PERsonalized MEdicine StRuggling for POLITICAL SUPPORT}

In the first decade of the new century, while scientific circles elaborated and refined the very idea of personalized medicine, politicians turned toward it as well. In particular, in 2006, then-Senator Barack Obama (D-IL), introduced a Genomics and Personalized Medicine Act intended to provide institutional support to the development of this field (S.3822).

The 2006 Act was the first of a series of four legislative initiatives bearing the same name and all aimed at fostering the promise of improving the accuracy of prevention, diagnosis and treatment. The other three were introduced in 2007 (S.976, again by Sen. Barack Obama, with support of Sen. Richard Burr, R-NC), in 2008 (H.R. 6498, by Congressman Patrick Kennedy, D-RI) and in 2010 (H.R. 5440, again by Kennedy and Congresswoman Anna Eshoo, D-CA), respectively. Despite growing expectations surrounding personalized medicine, however, none of these bills eventually passed into law. Examining them is nonetheless crucial for understanding how this field struggled to secure institutional endorsement, and what it took to eventually win that support in 2015.

\section{Genomics and Personalized Medicine Acts}

The first three versions of the Genomics and Personalized Medicine Act (2006, 2007 and 2008) share a similar definition of personalized medicine, intended as "the application of genomic and molecular data to better target the delivery of health care, facilitate the discovery and clinical testing of new products, and help determine a patient's predisposition to a particular disease or condition.'They also identify specific institutional needs that must be met in order to ensure personalized medicine fulfills its promise, including the need for federal leadership, the need to accelerate the rate of discovery, and incentives to data collection.

In terms of political coordination, those acts propose the creation of an Interagency Working Group within the Department for Health and Human Services (DHHS), charged with the aim of expanding and accelerating research and clinical translation by enhancing coordination and communication among Federal departments and agencies (sec. 4(1)(A)). 
The main stimulus to the growth of personalized medicine envisioned by those acts is the creation of the infrastructure that will accelerate the development of the field: a National Biobanking Distributed Database (NBDD) and Biobank Initiatives Grants (BIGs). NBDD is a system for the integration of sparse data to facilitate pooled analysis. BIGs, instead, serve the aim of fostering the establishment of biobanks throughout the country with the aim of advancing the application of genomics.

From an organizational perspective, in those acts research in personalized medicine relies on a distributed rather than a centralized model, leveraging resources from a variety of institutions disseminated across the country. Moreover, comprehensive and interoperable datasets constitute the central material asset for the further development of the field. Interestingly, as we have seen above, these two features would later be emphasized in the so-called P4 medicine model-thus signaling a two-way interaction between the political and the scientific discourse on personalized medicine.

Given the unsuccessful political fate of the first three acts, the fourth attempt, in 2010, had a substantively different framing. The very definition of the field appeared to be more comprehensive, now emphasizing "genes, environments and lifestyles" (emphasis added) as causally relevant factors of health, disease and prognosis.

In the new text, the main organizational change is the proposal to grant political coordination for the development of personalized medicine to an Office of Personalized Healthcare (OPH), within the office of the Secretary of the DHHS. The OPH, along with elaborating a long-term strategy of support for personalized medicine, is charged with the identification of the impediments to the translation of scientific discoveries into concrete clinical outputs, including obstacles related to unsuitable regulatory constraints.

The novelty of the infrastructure is its adoption of a centralized model. In particular, the act proposes the creation of a National Biobank under the aegis of the NIH that should be established to advance the field of genomics and personalized medicine. Grants for the promotion of local biobanking initiatives, however, are still present in the bill. This ambiguity as to whether to adopt a distributed or a centralized data and sample infrastructure may indicate that, at that stage, personalized medicine did not yet possess a well-defined organizational identity-a feature that may in part explain why this fourth attempt was also doomed to fail in Congress.

And yet, for the promoters of the Genomics and Personalized Medicine Acts, the development of this field needed to be backed up politically. More specifically, to keep up with its promises, the field needed a dedicated infrastructure whose creation was a responsibility of the government. The stimuli envisaged at the policy level, therefore, sought to create the conditions for the emergence of personalized medicine. But oscillations between a distributed and a centralized model for 
this infrastructure reveal that, despite grandiose claims about the expected impact of personalized medicine, ideas were less than clear as to how the whole plan was supposed to actually work in practice. Creating a distributed data infrastructure was initially envisaged as a priority. In the Genomics and Personalized Medicine Acts introduced between 2006 and 2008, the government was deeply implicated in the creation of a distributed database, while the collection of the biological samples from which data would emerge was delegated to local initiatives. However, such legislative initiatives were still very much focused on genetic data more than anything else. In scientific circles, the distributed model would enjoy a great deal of consideration until the end of the decade. By 2010, however, this model was no longer an obligatory passage point. In this last congressional attempt to secure support personalized medicine, emphasis shifts from a data to a specimens infrastructure-with the creation of a National Biobank.

This revised strategy anticipates a central feature of PMI: the creation of a precision medicine research cohort of one million Americans. Interestingly, the idea that data about individuals enabling the development of personalized therapies should be centrally collected first appears in a 2011 publication by the National Research Council entitled Toward Precision Medicine: Building a Knowledge Network for Biomedical Research and a New Taxonomy of Disease. Contrary to the PMI national research cohort, however, the Information Commons envisioned by the report is a database open for consultation to a wide spectrum of research and clinical actors and does not constitute a central repository of tissue samples. It is worth recalling that this report is also the first document from within the biomedical community to use the term "precision medicine," ${ }^{5}$ although the original use of this expression can be traced to a 2009 book about the application of disruptive innovation theory to healthcare (Christensen, Grossman, and Hwang 2009).

\section{The Political Upheaval of Precision Medicine}

And the goal of the Precision Medicine Initiative is to figure out how to break down some of the structural or institutional barriers that prevent us from making the big leaps over the next several years.

-President Barack Obama (White House 2016)

Against the backdrop of the historical trajectories we have identified thus far, in January 2015, President Obama announced the launch of a flagship plan to finally realize the promise of personalized therapy. In practical terms, the PMI distributes $\$ 215$ million from the President's budget to the NIH (\$130 million), the Nation-

\footnotetext{
${ }^{5}$ The report defines the "ultimate end point" of precision medicine as that of selecting "a subset of patients, with a common biological basis for disease, who are most likely to benefit from a drug or other treatment" (National Research Council 2011, 37). Successful examples of precision medicine, in the report, refer to pharmacogenomics approaches that are able to stratify disease taxonomies, such as the use of crizotinib in non-small cell lung cancer (38).
} 
al Cancer Institute (\$70 million), the FDA ( $\$ 10$ million), and the Office of the National Coordinator for Health Information Technology (\$5 million), in order to conduct a series of activities that should support the development of precision medicine. The goal of the initiative is ambitious, as it aims to "revolutionize how we improve health and treat disease” (White House 2015a).

Under the new banner of precision medicine, the White House designates "an innovative approach to disease prevention and treatment that takes into account individual differences in people's genes, environments, and lifestyles" (White House 2015a). The scope of this definition is reminiscent of earlier articulations of personalized medicine, or P4 medicine. However, the idea of considering disease from a systems perspective-as the perturbation of a baseline level of well-being - is not incorporated in precision medicine.

The envisioned goals of PMI are the acceleration of tailored cancer treatments, the constitution of a voluntary research cohort, protection of participants' privacy, regulatory modernization, and public-private partnership. Contrary to previous proposals, though, the initiative lacks a fixed center of political coordination and presents a more federalized structure, whereby each of the agencies involved will have to take care of a specific part of the project. The NIH, nonetheless, plays the most prominent role, both in terms of funding and in terms of the relative importance of its task. In particular, the NIH would immediately start assembling a large longitudinal research cohort comprised of at least one million individuals who will consent to be comprehensively characterized based on tissue samples, behavioral data drawn from portable devices (such as smartphones and wearable devices), and medical health records.

Of the aims of the cohort, some are more specifically scientific, such as developing risk estimates based on multiple data types, optimizing cancer treatments, identifying the determinants of safety and efficacy for commonly used drugs, discovering biomarkers for common diseases, and developing the use of mobile health technologies to predict health outcomes. Others however, are more aspirational in nature and incorporate a broader societal vision. In particular, the idea of engaging and empowering participants with their own data to use them to improve their health represents a distinctive cultural feature of the whole initiative. Interestingly, the research participant prefigured by the PMI research cohort is therefore both a contributor and an end user of the data. This feature aligns with the anticipated diversity of the data to be extracted from the PMI cohort. Recent renderings of precision medicine seem to have incorporated the idea that the development of the field is predicated upon such diversity. In particular, Hawgood and colleagues (2015) have argued that the integration of genomic data, microbiomic data, environmental exposure data, behavioral data, patients' health records, and participant-contributed data - even of the most unstructured form (like postings on social network or data uploaded from smartphone apps) — will bring precision medicine beyond the "inflection point" at which "dramatic change" can 
happen. Optimistic statements of this sort show that precision medicine is still characterized by the same aspirations that have marked its predecessors. Even so, the role that research participants might play in moving beyond that inflection point is a novel and distinctive feature of the PMI.

The PMI research cohort is imagined to rely on two modes of recruitment: direct volunteering and solicited enrollment during clinical interaction. While the latter mode coincides with the conventional enrollment strategy for clinical research, the former draws on the crowd-sourcing model originally envisaged by Hood and Friend (2011). Reminiscent of its participatory ethos-also initially developed by the proponents of P4 medicine- the PMI thus explicitly sets out to promote a cultural as well as a scientific revolution (PMI Working Group 2015), capitalizing on people's willingness "to be active partners in modern science" (Collins and Varmus 2015). Public engagement in the governance of the cohort is thus imagined to ensure that its activities respond to the expectations and values of those who contribute to it in the first person. The expected advantage of this partnership, as imagined in the PMI, consists in fostering collaboration between participants and researchers in the design and management of the cohort, thus ensuring trust and sustainability for the initiative (White House 2015b). The vision of the PMI in this domain, though, goes beyond issues of governance. In exchange for their participation, volunteers will also get access to the information generated about them through their data, thus being empowered to potentially use that information for health purposes. ${ }^{6}$

Precision medicine, therefore, does not only embrace a general trend towards expanding the scope of research participation. It also seeks to promote a culture of personal responsibility for one's own health, and does so by using the language of empowerment. But because terms like "participation" and "empowerment" carry positive connotations, we should be attentive the full spectrum of ethical implications - both positive and negative - that these terms might have on the public perception of precision medicine.

\section{ETHICAL AND CULTURAL DRIVERS OF BIOMEDICAL INNOVATION}

Our analysis has traced the presence of distinctive features in the framing of personalized medicine that eventually won political support for the program. With respect to previous scientific and legislative articulations of the idea of personalized medicine, the PMI embraces a centralized model of development and sets out to create an ad hoc data and biospecimens infrastructure. Moreover, accord-

\footnotetext{
${ }^{6}$ It remains to be seen if and to what extent this model will turn out to be empowering in terms of tangible health outcomes. Indeed, some commentators have expressed perplexities on the overall vision of precision medicine in this respect. Although interesting in their own rights, those critiques cannot be discussed here (see Bayer and Galea 2015).
} 
ing to the policy documents we have analyzed, public engagement - understood as active participation and empowerment-is a driving value of the enterprise. While we should remember to be cautious about the commendatory connotation that those values carry, here we are simply interested in describing the normative plan that the PMI promotes as a federally supported science policy initiative.

It is notable that in the 2016 PMI summit at the White House, President Obama's remarks reiterated the centrality of those positive values in several ways. Two passages in particular spelled out the cultural, not just the scientific, promise of precision medicine. First, the President described the core message of the initiative as "empowering individuals to monitor and take a more active role in their own health" (emphasis added). Second, the President remarked about the ethics of data ownership, by pointing out that creating a large precision medicine cohort "requires, first of all, us understanding who owns the data. And I would like to think that if somebody does a test on me or my genes, that's mine. But that's not always how we define these issues" (White House 2016).

Data ownership is a hotly debated issue, likely to affect the development of precision medicine in the years to come. A number of stakeholders, including participants, clinical institutions, sponsors, and researchers and their funders, all claim some kind of ownership over the data collected and generated in the course of research. Strong arguments on both sides critically bear on questions of empowerment and partnership (Terry and Terry 2011). Unsurprisingly, then, President Obama's remark suggests the importance of attributing ownership to engaged participants who contribute samples and data.

This emphasis on the ethical value of public engagement, partnership, and empowerment is noteworthy in the legislative initiatives for personalized medicine. However, the emphasis on ethics is familiar in the broader context of discussions concerning biotechnology and genomic research in the last decade. In genomics, patients' advocacy groups have long been championing a movement to shift data ownership and control to participants who actually contribute those data (Terry and Terry 2011). In particular, those movements are interested in promoting participants' sense of empowerment and participation by granting them a wide array of options concerning the selection of where their data should go, who should have access to the data, and how the data should be used. Allowing people to share their data, many patients' advocates insist, will allow clinical trial data to circulate widely, thus boosting the odds of scientific discovery.

Appeals to these understandings have become a defining feature of precision medicine. Within this context, the idea of "patient engagement" has been described as "the next blockbuster drug," precisely because it offer patients the tools to become actively involved with their health (Chase 2016). Involving patients and the public more broadly in science and clinical matters has gained momentum in discussions of regulating the increasingly complex challenges of risk management in biotechnology (Irwin 2006; Solomon, Gusmano, and Maschke 2016). 
Participant-centered governance initiatives have also gained widespread recognition with international research consortia that collect and analyze extensive datasets (Kaye et al. 2012). With large-scale biobanking research activities, participants' engagement as partners of research can now be considered a central component of mainstream ethical frameworks (Blasimme and Vayena 2016).

Notably, these values are also being explicitly articulated in the private sector. In particular, the personal genomic industry has grown around a narrative of empowerment. Pioneering players in this sector, like 23andMe, have long been selling genomic testing to consumers based on the idea that consumers can be empowered to freely make choices about their health based on the test results, without invention from their physicians (Prainsack and Vayena 2013; Vayena 2014). As the NIH develops an infrastructure for precision medicine centered around a 1 million participant research cohort, 23andMe already possesses samples and SNPs data from 1.5 million customers, revealing the capacity of the private sector to shape the development of big data health research by leveraging both cultural and material resources.

Framing precision medicine with the core ethical values of public engagement, partnership, and empowerment aligns the model with prominent normative narratives characterizing genetics, genomics, and biobanking research. This ethical framework may have been a major driver in ensuring federal support for precision medicine. Despite widespread enthusiasm for the ethos of participation, however, the idea of empowerment has also attracted some pointed criticism. Eric Juengst and colleagues, for example, point out that while a rhetoric of empowerment is gaining ground in federal policy, it raises a risk of "a relocation of responsibility for health care away from social and political realms and onto the shoulders of the patients" themselves (Juengst, Flatt, and Settersten 2012, 36). Obviously, it is too early to know whether this risk will actually materialize as precision medicine moves forward. Nevertheless, we need to pay attention to how a driving normative ideal centered around empowerment might take shape in practice, and what its ethical tradeoffs might be.

\section{CONCLUSION}

The creation of a large-scale centralized cohort with millions of participants represents the visible material incarnation of precision medicine as a novel federally supported biomedical model. The initiative is also unusual with respect to how research participants and patients involved in its development are to be considered, how they will be involved, and how they are to be treated. Precision medicine incorporates a central role for patients and research participants understood as "active partners in clinical research" (Collins and Varmus 2015). In part by taking ethical issues seriously, the initiative has received a remarkable level of institutional and political support. 
The launch of PMI shows how specific values can become embedded in the public articulation of biomedical programs. Careful examination of the process can clarify how innovations are granted or denied political recognition and support. Scientific progress and political usefulness alone are not sufficient to explain the success of precision medicine as a federally supported initiative. Weaving together the scientific and legislative antecedents of precision medicine illuminates how the mutual constitution of science and ethical values can generate political recognition for an innovative biomedical paradigm (Jasanoff 2005).

\section{REFERENCES}

Bayer, R., and S. Galea. 2015. "Public Health in the Precision-Medicine Era." N Engl J Med 373 (6): 499-501. doi:10.1056/NEJMp1506241.

Biomarkers Definitions Working Group. 2001. "Biomarkers and Surrogate Endpoints: Preferred Definitions and Conceptual Framework." Clin Pharmacol Ther 69 (3): 89-95. doi:10.1067/mcp.2001.113989.

Blasimme, A. and E. Vayena. 2016. "Becoming Partners, Retaining Autonomy: Ethical Considerations on the Development of Precision Medicine." BMC Medical Ethics 17:67. Chase, D. 2016. "Patient Engagement Is the Blockbuster Drug of the Century." Forbes, Sept. 9. http://www.forbes.com/sites/davechase/2012/09/09/patient-engagement-isthe-blockbuster-drug-of-the-century/.

Chin, L., J. N. Andersen, and P. A. Futreal. 2011. "Cancer Genomics: From Discovery Science to Personalized Medicine.” Nat Med 17 (3): 297-303.

Christensen, C. M., J. H. Grossman, and J. Hwang. 2009. The Innovator's Prescription: A Disruptive Solution for Health Care. New York: McGraw-Hill.

Collins, F. S., and H.Varmus. "A New Initiative on Precision Medicine.” N Engl J Med 37 (9): 793-95.

Evans, W. E., and M.V. Relling. 1999. "Pharmacogenomics: Translating Functional Genomics into Rational Therapeutics." Science 286 (5439): 487-91.

Evans, W. E., and M. V. Relling. 2004. "Moving Towards Individualized Medicine with Pharmacogenomics." Nature 429: 464-68.

Flores, M., et al. 2013. "P4 Medicine: How Systems Medicine Will Transform the Healthcare Sector and Society." Pers Med 10 (6): 565-76.

Gardiner, S. J., and E. J. Begg. 2006. "Pharmacogenetics, Drug-Metabolizing Enzymes, and Clinical Practice.” Pharmacol Rev 58 (3): 521-90.

Ginsburg, G. S., and J. J. McCarthy. 2001. "Personalized Medicine: Revolutionizing Drug Discovery and Patient Care." Trends Biotechnol 19 (12): 491-96. doi:10.1016/S01677799(01)01814-5.

Ginsburg, G. S., and H. F. Willard. 2009. "Genomic and Personalized Medicine: Foundations and Applications." Transl Res 154 (6): 277-87.

Gonzalez, F. J. 1988. "The Molecular Biology of Cytochrome P450s.” Pharmacol Rev 40 (4): 243-88.

Hamburg, M. A., and F. S. Collins. 2010. “The Path to Personalized Medicine.” N Engl J Med 363 (4): 301-4. 
Hawgood, S., et al. 2015. "Precision Medicine: Beyond the Inflection Point." Sci Transl Med 7 (300): 300ps17. doi:10.1126/scitranslmed.aa99970.

Hood, L., and M. Flores. 2012."A PersonalView on Systems Medicine and the Emergence of Proactive P4 Medicine: Predictive, Preventive, Personalized and Participatory." New Biotechnol 29 (6): 613-24.

Hood, L., and S. H. Friend. 2011. "Predictive, Personalized, Preventive, Participatory (P4) Cancer Medicine." Nat Rev Clin Oncol 8 (3): 184-87.

Irwin, A. 2006. "The Politics of Talk Coming to Terms with the 'New' Scientific Governance." Soc Stud Sci 36 (2): 299-320. doi:10.1177/0306312706053350.

Jasanoff, S. 2004. "Ordering Knowledge, Ordering Society." In States of Knowledge: The Co-Production of Science and the Social Order, ed. S. Jasanoff, 13-45. London: Routledge.

Jones, D. S. 2013. “The Prospects of Personalized Medicine.” In Genetic Explanation: Sense and Nonsense, 147-70. Cambridge: Harvard University Press.

Juengst, E.T., M.A. Flatt, and R.A. Settersten. 2012. "Personalized Genomic Medicine and the Rhetoric of Empowerment." Hastings Cent Rep 42 (5): 34-40.

Kaye, J., et al. 2012. "From Patients to Partners: Participant-Centric Initiatives in Biomedical Research." Nat Rev Genet 13 (5): 371-76.

Kitano, H. 2002. “Systems Biology: A Brief Overview.” Science 295 (5560): 1662-64.

Meyer, U. A. 2000. "Pharmacogenetics and Adverse Drug Reactions.” Lancet 356 (9242): 1667-71.

National Research Council. (2011). Committee on A Framework for Developing a New Taxonomy of Disease. Toward Precision Medicine: Building a Knowledge Network for Biomedical Research and a New Taxonomy of Disease. Washington, DC: National Academies Press.

Nicholson, J. K. 2006. "Global Systems Biology, Personalized Medicine and Molecular Epidemiology.” Mol Syst Biol 2 (1). doi:10.1038/msb4100095.

Prainsack, B., and E. Vayena. 2013. "Beyond the Clinic: 'Direct-to-Consumer' Genomic Profiling Services and Pharmacogenomics.” Pharmacogenomics 14 (4): 403-12.

Precision Medicine Initiative (PMI) Working Group. 2015. The Precision Medicine Cohort Program: Building a Research Foundation for 21st Century Medicine. NIH. http://acd. od.nih.gov/reports/DRAFT-PMI-WG-Report-9-11-2015-508.pdf.

Roses, A. D. 2000. "Pharmacogenetics and the Practice of Medicine." Nature 405 (6788): 857-65.

Roses, A. D. 2004. "Pharmacogenetics and Drug Development: The Path to Safer and More Effective Drugs." Nat Rev Genet 5 (9): 645-56.

Solomon, M. Z., M. K. Gusmano, and K. J. Maschke. 2016. "The Ethical Imperative and Moral Challenges of Engaging Patients and the Public with Evidence." Health Aff (Millwood) 35 (4): 583-89. doi:10.1377/hlthaff.2015.1392.

Steele, F. R. 2009. "Personalized Medicine: Something Old, Something New." Pers Med 6 (1): $1-5$.

Terry, S. F., and P. F. Terry. 2011. "Power to the People: Participant Ownership of Clinical Trial Data." Sci Transl Med 3 (69): 69cm3.

U.S. Food and Drug Administration (FDA). 2016. Table of Pharmacogenomic Biomarkers in Drug Labeling. http://www.fda.gov/Drugs/ScienceResearch/ResearchAreas/ Pharmacogenetics/ucm083378.htm. 
Vayena, E. 2014. "Direct-to-Consumer Genomics on the Scales of Autonomy." J Med Ethics 41 (4): 310-14. doi:10.1136/medethics-2014-102026.

Weston, A. D., and L. Hood. 2004. "Systems Biology, Proteomics, and the Future of Health Care:Toward Predictive, Preventative, and Personalized Medicine." J Proteome Res 3 (2): 179-96.

Whirl-Carrillo, M., et al. 2012. "Pharmacogenomics Knowledge for Personalized Medicine." Clin Pharmacol Ther 92 (4): 414-17. doi:10.1038/clpt.2012.96.

White House. 2015a. Fact Sheet: President Obama's Precision Medicine Initiative. https://www.whitehouse.gov/the-press-office/2015/01/30/fact-sheet-president-obama-s-precision-medicine-initiative.

White House. 2015b. Privacy and Trust Principles for the President's Precision Medicine Initiative. https://www.whitehouse.gov/sites/default/files/microsites/finalpmiprivacyandtrustprinciples.pdf.

White House. 2015c. Remarks by the President on Precision Medicine. https://www. whitehouse.gov/the-press-office/2015/01/30/remarks-president-precision-medicine.

White House. 2016. Remarks of the President in Precision Medicine Panel Discussion. 2016. https://www.whitehouse.gov/the-press-office/2016/02/25/remarks-presidentprecision-medicine-panel-discussion. 